\title{
Medaka Melanoma
}

National Cancer Institute

\section{Source}

National Cancer Institute. Medaka Melanoma. NCI Thesaurus. Code C134572.

Melanoma that occurs in Oryzias latipes. 